\title{
AN ELECTRICAL CIRCUIT THEORETICAL METHOD FOR TIME- AND FREQUENCY-DOMAIN SOLUTIONS OF THE STRUCTURAL MECHANICS PROBLEMS
}

\author{
A. SUAT EKINCI* AND ABDULLAH ATALAR \\ Department of Electrical and Electronics Engineering, Bilkent University, 06533, Ankara, Turkey
}

\begin{abstract}
SUMMARY
Shrinking device dimensions in integrated circuit technology made integrated circuits with millions of components a reality. As a result of this advance, electrical circuit simulators that can handle very large number of components have emerged. These programs use new circuit simulation techniques and can find solutions accurately and quickly. In this paper, we apply these techniques to structural mechanics problems by adopting electrical circuit equivalents. We first apply finite element formulation to the mechanical problem. The obtained sets of equations are treated as if they are sets of equations of an equivalent electrical circuit which consists of linear circuit elements such as capacitors, inductors and controlled sources. The equivalent circuit is obtained in the form of a circuit netlist and solved using a general purpose electrical circuit simulator. Several examples showing the advantages of the circuit simulation techniques are demonstrated. Asymptotic waveform evaluation technique which is widely used for simulation of large electrical circuits is also studied for the same examples and the speed-up advantage is shown. Copyright (C) 1999 John Wiley \& Sons, Ltd.
\end{abstract}

KEY WORDS: asymptotic waveform evaluation (AWE); circuit simulation; finite element modelling; structural mechanics; electrical modelling of mechanical systems

\section{INTRODUCTION}

Finite element method, which is used for finding an approximate solution for problems, has found extensive applicability in the field of structural mechanics. This method has now become a predominant analysis and design tool.

Finite element formulations result in large sets of equations. Space/frequency formulations involve the solution of the large system at many frequency points or require the computationally expensive process of determining eigenvalues and corresponding eigenvectors of large matrices.

In both structural mechanics problems and electrical circuit simulation problems we meet similar types of analyses; namely steady-state (or time-independent) analysis, eigenvalue analysis, and propagation (or transient) analysis.

The electrical circuit simulation programs employ different methods to find the circuit behaviour. Spice-like programs [1-3] are used for intensive verification of large circuits and find high-accuracy solutions. In these programs the inverse of the matrix is calculated at a large number of points in

* Correspondence to: A. Suat Ekinci, Department of Electrical and Electronics Engineering, Bilkent University, 06533, Bilkent, Ankara, Turkey. E-mail: ekinci@ee.bilkent.edu.tr

CCC 0029-5981/99/221485-23\$17.50

Received 14 January 1998

Copyright (C) 1999 John Wiley \& Sons, Ltd.

Revised 3 November 1998 
both frequency and time domains. However, most of the new simulators involve smaller number of matrix solutions.

Asymptotic Waveform Evaluation [4] (AWE) technique is widely used in the simulation of very large circuits. In this method the system behaviour is approximated with a lower-order model. The method is based on the Taylor series expansion of the circuit response around $s=0$, and is very efficient to extract the low-frequency behaviour of the circuit.

To find the behaviour in a frequency range of interest which does not contain only low-frequency regions, Complex Frequency Hopping (CFH) technique is introduced [5]; and recently a multi-point Padé-approximation technique is developed $[6,7]$. In these studies, the expansions for different frequency points are found. The multi-point moment matching method may also be extended to use the information of expansions at infinity [8]. This information is basically needed to approximate the transient behaviour.

Several studies have been done to use the simplicity of the circuit solution techniques in the field analysis problems. Kron [9] suggested equivalent circuits of the elastic fields, and Carter [10] dealt with the solution techniques of these circuits. In those circuits, the stresses are represented by currents and the strains by voltages. Electrical equivalents of the equations are obtained and satisfied by the equivalent networks.

In the recent years, electrical thermal network analogy is widely used to study thermal behaviour of electronic components, and the analogy leads to large Resistor-Capacitor (RC) networks which are analysed by using circuit simulation techniques. In the work of Hsu et al. [11] elemental thermal circuit networks, which correspond to elements in 1-D, 2-D and 3-D cases are developed. These networks are connected to the electrical networks to provide complete electrothermal models that can be used in any circuit simulation packages. Same authors also studied model reduction techniques for large problems [12].

There are studies on coupling the external circuit equations with finite element models. These studies can be classified into two different approaches: the equations of the finite element model and circuit model may be handled as a single system of equations [13-17], or finite element part may be handled as a separate system which communicates with the circuit model [18-21]. The first approach is called direct coupling and the second is called indirect coupling. Direct coupling is popular, because quite effective and reliably convergent computation is possible by applying Newton-Raphson iteration on the combined problem. Usually the number of nodes in finite element mesh is so large that sparse matrix methods have to be used, but the combination of the models change the sparsity and symmetry of the finite element matrices. In indirect coupling, the finite element model is handled separately [19-21]. In the works of Väänänen [19] and McDermott et al. [21] the parts modelled by the finite element method are considered as a multiport element in the circuit. Both of these works require the solution of the finite element model first.

Transmission line modelling [22] is also used in stress-strain analysis problems, but it is difficult to handle the analysis of transmission lines. Another disadvantage of such modelling is the introduction of voltage source inductor loops which have to be solved using special techniques. AWE technique in circuits containing transmission lines is not as efficient as in the RLC circuits because of the extra multiplications in moment updating procedure.

Recently, techniques mainly developed for circuit simulation are used in the solution of electromagnetic analysis problems. AWE is proposed in the study by Gong [23], where the reduction of the order of the parameters, such as input impedance, $S$ parameters, and far-field pattern using a new moment update procedure is discussed. In the work of Kolbehdari, $\mathrm{CFH}$ technique is studied [24]. In CFH technique frequency range is divided into regions and the response is approximated 
by different transfer functions at each frequency region. Both studies show that usage of circuit simulation techniques is promising.

In this paper, the analogy between the FEM solutions of the structural problems and the electrical circuit simulation problems is presented. A technique to extract the equivalent circuit for a structure is introduced, and the speed-up advantage obtained using general purpose electrical circuit simulators HSPICE [3] and MAWE [7] is demonstrated.

\section{FORMULATION}

In a typical structural analysis, we try to find the stress and displacement distribution in a rigid body under a set of loading and boundary conditions. The following equations have to be solved to find an analytical solution of the problem.

$$
\begin{aligned}
\tilde{\nabla}_{s} \cdot \mathbf{T} & =\rho \frac{\partial \mathbf{v}}{\partial t}-\mathbf{F} \\
\mathbf{T} & =\mathbf{c}: \mathbf{S} \\
\mathbf{S} & =\nabla_{s} \mathbf{u}
\end{aligned}
$$

In these equations and throughout the paper the following notation is used: $\mathbf{u}$ is the displacement vector $\left(u_{x}, u_{y}, u_{z}\right), \mathbf{v}$ the velocity vector $\left(v_{x}, v_{y}, v_{z}\right), \mathbf{S}$ the Strain tensor $\left(S_{1}=S_{x x}, S_{2}=S_{y y}, S_{3}=S_{z z}\right.$, $\left.S_{4}=S_{y z}, S_{5}=S_{x z}, S_{6}=S_{x y}\right), \mathbf{T}$ the Stress tensor $\left(T_{1}=T_{x x}, T_{2}=T_{y y}, T_{3}=T_{z z}, T_{4}=T_{y z}, T_{5}=T_{x z}\right.$, $\left.T_{6}=T_{x y}\right)$, c the elastic stiffness matrix and $\rho$ the mass density.

In the equations ' $\nabla_{s}$ ' corresponds to an operator represented by the following matrix:

$$
\nabla_{s} \rightarrow\left[\begin{array}{ccc}
\partial / \partial x & 0 & 0 \\
0 & \partial / \partial y & 0 \\
0 & 0 & \partial / \partial z \\
0 & \partial / \partial z & \partial / \partial y \\
\partial / \partial z & 0 & \partial / \partial x \\
\partial / \partial y & \partial / \partial x & 0
\end{array}\right]
$$

and ' $\tilde{\nabla}_{s} \cdot$ ' corresponds to the operator represented by the transpose of the same matrix. Velocity, the time derivative of displacement; i.e. $v=\partial u / \partial t$, is also used. Table I shows the equations and unknowns.

Table I. Equations (1)-(3) and unknowns in structural problems

\begin{tabular}{lccc}
\hline Type of equations & $\begin{array}{c}\text { Number of } \\
\text { equations }\end{array}$ & Unknowns & $\begin{array}{c}\text { Number of } \\
\text { unknowns }\end{array}$ \\
\hline $\begin{array}{l}\text { Translational eqn. of motion } \\
\text { Stress-strain relation }\end{array}$ & 3 & Displacement & 3 \\
$\begin{array}{l}\text { Strain-displacement } \\
\text { relations }\end{array}$ & 6 & Stress & 6 \\
Total & 6 & Strain & 6 \\
\hline
\end{tabular}


In practice, equations known as compatibility equations (continuity of strains and displacements), and boundary conditions (conditions on displacements, forces at the boundary) also have to be satisfied.

\subsection{Finite element method}

Finite element method is used to find an approximate solution of the problem. In the literature, displacement method or the minimum potential approach have been extensively used. In this method, all the equations are written in terms of the displacement components and solved for unknown displacements. We will give a brief flow of algorithm [25-27] for the dynamical systems in order to make the analogy between the structural problems and the electrical circuit problems clear.

Let the body be meshed into $E$ elements. The displacement model of the eth element is denoted as:

$$
\vec{u}^{(e)}(x, y, z, t)=\left\{\begin{array}{l}
u_{x}^{(e)}(x, y, z, t) \\
u_{y}^{(e)}(x, y, z, t) \\
u_{z}^{(e)}(x, y, z, t)
\end{array}\right\}=\mathbf{N}(x, y, z) \vec{Q}^{(e)}(t)
$$

where $\vec{u}^{(e)}$ is the vector of displacement, $\mathbf{N}$ is the matrix of shape functions, $\vec{Q}^{(e)}$ is the vector of degrees of freedom and is assumed to be a function of time $t$.

Using (3) with $\mathbf{B}=\nabla_{s} \mathbf{N}$, the strains can be approximated as

$$
\mathbf{S}^{(\mathbf{e})}=\mathbf{B} \vec{Q}^{(e)}
$$

and stresses as

$$
\mathbf{T}^{(\mathbf{e})}=\mathbf{c}: \mathbf{S}^{(\mathbf{e})}=\mathbf{c B} \vec{Q}^{(e)}
$$

The kinetic energy $\pi_{\mathrm{k}}$ and the potential energy $\pi_{\mathrm{p}}$ can be expressed as:

$$
\pi_{\mathrm{k}}=\sum_{e=1}^{E} \pi_{\mathrm{k}}^{(e)}=\frac{1}{2} \dot{\overrightarrow{\mathbf{Q}}}^{\mathrm{T}}\left[\sum_{e=1}^{E} \iiint_{V^{(e)}} \rho \mathbf{N}^{\mathrm{T}} \mathbf{N} \mathrm{d} V\right] \dot{\overrightarrow{\mathbf{Q}}}
$$

where $[\cdot]^{\mathrm{T}}$ denotes the transpose of the matrices and the vectors.

$$
\pi_{\mathrm{p}}=\sum_{e=1}^{E} \pi_{\mathrm{p}}^{(e)}=\frac{1}{2} \overrightarrow{\mathbf{Q}}^{\mathrm{T}}\left[\sum_{e=1}^{E} \iiint_{V^{(e)}} \mathbf{B}^{\mathrm{T}} \mathbf{c} \mathbf{B} \mathrm{d} V\right] \overrightarrow{\mathbf{Q}}-\overrightarrow{\mathbf{Q}}^{\mathrm{T}} \overrightarrow{\mathbf{P}}(t)
$$

where $\overrightarrow{\mathbf{P}}(t)$ is the total load vector. Matrices $\mathbf{M}$, master mass matrix of the structure and $\mathbf{K}$, master stiffness matrix of the structure, are defined by

$$
\begin{aligned}
\mathbf{M} & =\sum_{e=1}^{E} \iiint_{V^{(e)}} \rho \mathbf{N}^{\mathrm{T}} \mathbf{N} \mathrm{d} V \\
\mathbf{K} & =\sum_{e=1}^{E} \iiint_{V^{(e)}} \mathbf{B}^{\mathrm{T}} \mathbf{c} \mathbf{B} \mathrm{d} V
\end{aligned}
$$

Using a modified form of Lagrange's equations [28],

$$
\frac{\mathrm{d}}{\mathrm{d} t}\left\{\frac{\partial L}{\partial \dot{Q}_{i}}\right\}-\frac{\partial L}{\partial Q_{i}}+\frac{\partial R}{\partial \dot{Q}_{i}}=0, \quad i=1,2, \ldots, n
$$


where $L=\pi_{\mathrm{k}}-\pi_{\mathrm{p}}$ is the Lagrangian, $n$ is the number of unknowns and $R$ stands for the dissipation. We obtain the desired dynamic equations of motion of the structure.

$$
\mathbf{M} \ddot{\overrightarrow{\mathbf{Q}}}(t)+\mathbf{C} \dot{\overrightarrow{\mathbf{Q}}}(t)+\mathbf{K} \overrightarrow{\mathbf{Q}}(t)=\overrightarrow{\mathbf{P}}(t)
$$

C is the master damping matrix of the structure, and it is usually defined as

$$
\mathbf{C}=\alpha \mathbf{M}+\beta \mathbf{K}+\mathbf{C}_{\xi}
$$

where $\alpha$ and $\beta$ are constants, and $\mathbf{C}_{\xi}$ is the frequency-dependent damping matrix. If damping is neglected, we have the lossless case:

$$
\mathbf{M} \ddot{\overrightarrow{\mathbf{Q}}}(t)+\mathbf{K} \overrightarrow{\mathbf{Q}}(t)=\overrightarrow{\mathbf{P}}(t)
$$

Equations (11) and (13) can be solved using different mathematical techniques, once the vector $\overrightarrow{\mathbf{Q}}(t)$ is known, the variations of the displacements, stresses and strains in the elements can be found.

Various kinds of analysis of dynamical systems can be done using the equation of motion.

If the aim of the stress analysis or solid mechanics problems is to find the distribution of displacements and stresses under the stated loading and boundary conditions, there will be no time dependency, so the equation becomes

$$
\mathbf{K} \overrightarrow{\mathbf{Q}}=\overrightarrow{\mathbf{P}}
$$

In transient analysis all the unknowns are time dependent, and the equation of motion has to be solved. Generally, the finite difference method or Newmark's method is used to find the solution.

The frequency behaviour of the structure can be found using harmonic analysis. The displacements are assumed to be harmonic as

$$
\overrightarrow{\mathbf{Q}}(t)=\overrightarrow{\mathbf{Q}} \cdot \mathrm{e}^{\mathrm{j} \omega t}
$$

where $\mathrm{j}=\sqrt{-1}$ and the equation becomes

$$
\left[-\omega^{2} \mathbf{M}+\mathbf{j} \omega \mathbf{C}+\mathbf{K}\right] \overrightarrow{\mathbf{Q}}=\overrightarrow{\mathbf{P}}
$$

The oscillatory motion occurs at certain frequencies known as natural frequencies or characteristic values, and follows well-defined deformation patterns known as mode shapes or characteristic modes. The natural frequencies and mode shapes can be found using eigenvalue analysis techniques. The modes are the eigenvalues of the system defined by equation (14).

\subsection{Electrical circuit simulation}

For linear electrical circuits the state equations are written as follows [29]:

$$
\mathbf{C} \dot{x}=-\mathbf{G} x+\mathbf{b} u(t)
$$

where $\mathbf{C}$ is the matrix of capacitances and inductors, $\mathbf{G}$ is the admittance matrix, $u(t)$ is the voltage or current excitation at the nodes defined by vector $\mathbf{b}$, and the unknown state vector $x$ contains nodal voltages, inductor currents and voltage supply currents. The set of the equations come from the modified node analysis. In the appendix modified node analysis formulation is described, and 
the current voltage relations of the electrical circuit components which we use in this paper are shown.

In transient analysis, time variations of the state variables, the entries of the vector $x$, are found. $\mathrm{In} \mathrm{AC}$ analysis the frequency response of the system is investigated. i.e. the equation

$$
(\mathbf{G}+s \mathbf{C}) X(s)=\mathbf{b} U(s)
$$

is solved. Frequency response is obtained at $s=\mathrm{j} \omega(\mathrm{j}=\sqrt{-1})$.

In pole-zero analysis, the eigenvalues of the system defined by equation (16) are found. The dynamic equation of motion (11) which is a second-order differential equation can be converted into an equation of order one [26], so that it will be the same as the circuit equation (15) and it can be solved using circuit simulation techniques.

The shrinking of the circuit sizes makes it possible to build larger circuits which results in large systems of equations. Since the direct solutions of such systems will take a lot of time, the circuit simulation techniques are improved in order to handle the analysis. New methods for faster analysis are developed without losing the accuracy.

\subsection{Overview of moment-matching techniques}

The moment-matching techniques are widely used in circuit simulation in order to reduce the execution time [4-7, 30-34]. In these techniques by approximating the dominant poles of the circuit with a lower-order model, the behaviour of the circuit is obtained.

Moment-matching uses the coefficients (moments) of the expansion of the system transfer function, $H(s)$, around a point in the complex $s$-plane. The Taylor series expansion of $H(s)$ around $s_{0}$ is given as:

$$
H(s)=m_{0}+\left(s-s_{0}\right) m_{1}+\left(s-s_{0}\right)^{2} m_{2}+\cdots
$$

After the moments are generated, they are matched to a ratio of two polynomials $[4,6,7]$ or to a low-order set of poles and residues [5] by using Padé approximation.

2.3.1. Generation of the moments. The transfer function for a circuit which consists of lumped elements is (using equation (16))

$$
H(s)=(\mathbf{G}+s \mathbf{C})^{-1} \mathbf{b}
$$

or with a change of variable $s=s_{0}+\sigma$

$$
H\left(s_{0}+\sigma\right)=\left(\mathbf{I}+\sigma\left(\mathbf{G}+s_{0} \mathbf{C}\right)^{-1} \mathbf{C}\right)^{-1}\left(\mathbf{G}+s_{0} \mathbf{C}\right)^{-1} \mathbf{b}
$$

The transfer function is approximated as

$$
\hat{H}(s)=\left(\mathbf{I}+\sigma \mathbf{A}+\sigma^{2} \mathbf{A}^{2}+\cdots+\sigma^{n} \mathbf{A}^{n}\right) \mathbf{r}
$$

where

$$
\begin{aligned}
s & =s_{0}+\sigma \\
\mathbf{A} & =-\left(\mathbf{G}+s_{0} \mathbf{C}\right)^{-1} \mathbf{C} \\
\mathbf{r} & =\left(\mathbf{G}+s_{0} \mathbf{C}\right)^{-1} \mathbf{b}
\end{aligned}
$$


As a result the moments are found using $m_{n}=\mathbf{A}^{n} \mathbf{r}$. During the generation of moments the LU decomposition of the circuit matrix is calculated once for the first moment. Other moments are obtained using forward and backward substitutions.

In transient analysis, Laurent series expansion $(s=\infty)$ may also be used $[5,31-33,35]$. These moments are called the derivative moments, because they are the derivatives of the time response $[32,34]$.

2.3.2. $A W E, C F H$ and $M A W E$. In asymptotic waveform evaluation technique, to approximate the behaviour of the circuit, the Taylor expansion around $s=0$ is evaluated. Since the information carried by the moments is accurate at low-frequency region, the AWE technique is efficient in extracting the low-frequency poles of the circuit. At relatively higher frequencies the AWE technique becomes inefficient and several methods are proposed to improve the accuracy of AWE.

After the moments are generated, the transfer function is approximated with a rational function.

$$
\begin{aligned}
\hat{H}(s) & =m_{0}+m_{1} s+m_{2} s^{2}+\cdots+m_{n} s^{n} \\
& =\frac{B(s)}{A(s)}=\frac{\sum_{i=0}^{p} b_{i} s^{i}}{\sum_{j=0}^{q} a_{j} s^{j}} \\
& =\sum_{i=1}^{q} \frac{k_{i}}{s-p_{i}}
\end{aligned}
$$

where $p_{i}$ 's are the poles and $k_{i}$ 's are the corresponding residues. The methods to calculate the coefficients are documented in literature [4, 30,33]. First the coefficients $a_{i}$ are found by setting up a moment matrix, then poles and finally residues are calculated.

When the moments at $s=\infty$ are also included, a single moment matrix is set up again, and solved for the poles. Therefore the method is referred to as single-point Padé approximation.

Recently, multi-point Padé approximation techniques were proposed [5-7]. In the work by Chiprout et al. [5] Complex Frequency Hopping (CFH) technique is introduced. The transfer function $H(s)$ is expanded around $s=0$ and $s=\mathrm{j} \omega_{\max }$, and a binary search algorithm is employed until two neighbour expansions have a pole in common. Using these common poles, a region of accuracy is assigned for each expansion point. The poles in the region of accuracy and their corresponding residues are marked as accurate. The final estimate of transfer function contains all the marked poles and residues.

In the works of Celik et al. [6] and Sungur et al. [7] moment matching is done by simultaneously solving all the moment matrices obtained at each expansion. MAWE circuit simulation tool uses this method.

In finding circuit transfer functions, we usually choose $p=q-1$ in equation (20). This rational approximation is referred to as Padé approximation of order $q$. The method that we use for finding the coefficients, $a_{i}$ and $b_{i}$, is described in detail in the study by Sungur et al. [7].

The multipoint Padé approximation works not only for low frequencies, but also in highfrequency regions. Apart from the moments at $s=0$ (DC), the use of shifted moments provides the necessary information about the frequency range of interest. This approach requires the solution of the circuit matrix at several frequency points.

Padé approximation technique is very efficient in transient analysis. After obtaining the poles and residues of the system, the inverse transform; i.e. impulse response can be found easily.

When the number of poles in the problem is large, the approximation order should be large. This can be achieved by increasing the number of calculated moments, but this may give ill-conditioned 
moment matrices. To have a stable solution, $8-16$ moments have to be evaluated at each expansion point. Therefore, higher-order approximations can be reached by increasing the expansion points which means increasing the number of LU decompositions.

\section{EQUIVALENT CIRCUIT EXTRACTION}

Equivalent circuit approach is used to simulate the behaviour of a system with a simpler model or to solve coupled field-circuit problems. There are recent studies on electrothermal [11], electromechanical [21] and electromagnetic [19] problems which present procedures to build equivalent circuits.

In the work of McDermott et al. [21] the behavioural model of the electromechanical devices is extracted from a set of parametric finite element solutions. A new circuit element (a piecewise linear dependent source) is introduced. This component uses a look-up table produced by the FEM solutions. For example, given position and current, it returns flux and force.

In the work of Väänänen [19] the finite element model is handled as a circuit theoretical multiport element. In the FE model, unknowns other than the coupled potentials and currents are eliminated and a matrix equation for these electrical unknowns is derived. The problem is discretized in time so that the solution of a time-dependent problem is converted into successive solutions of a DC problem. The final equations are treated as electrical circuit equations and the FE model is represented by a multiport element containing Current Controlled Current Sources (CCCS), Voltage Controlled Current Sources (VCCS), Voltage Controlled Voltage Sources (VCVS) and Current Controlled Voltage Sources (CCVS). This method can handle non-linearities by updating the multiport electrical circuit parameters at each iteration.

The methods mentioned in the two works require the solution of FE model which takes a lot of time. However, in this study we want to demonstrate that the efficiency of the electrical circuit simulation techniques can be applicable to FE models.

In the study performed by Hsu et al. [11], thermal circuit networks, which are equivalent to discretization of the heat equation by FEM, are developed. Elemental thermal circuit networks are constructed for 1-D, 2-D and 3-D elements. The semidiscrete heat equation (continuous in time) derived from a Galerkin finite element projection [11] is

$$
\mathbf{M d}+\mathbf{K d}=\mathbf{F}
$$

It is easily seen that the mass matrix $\mathbf{M}$ can be realized using capacitor elements, the stiffness matrix $\mathbf{K}$ by resistor elements and the force vector $\mathbf{F}$ by current sources. The procedure for deriving the equivalent circuits is given in the article. The simulations were performed using SABER [36] circuit simulator where the thermal networks are written as element templates. This method is useful for field-circuit coupled problems, e.g. simulation of self-heating effects in semiconductor devices.

The works summarized up to now are suggested to solve the coupled field-circuit problems. While the first two methods require the simulation of the FE model, throughout the procedure in the last method no matrix inversion, or decomposition is needed. However, this method may introduce negative values for capacitances and resistors, and this is not acceptable in a general circuit simulation tool. In this study, we propose a method which does not need any matrix inversion in the circuit construction procedure. The final circuit can be solved using a general circuit simulator, and fast methods developed for simulation of electrical circuits can be used. 
The method suggested by Hsu et al. [11] can be generalized for use with second-order equations. One can realize matrices $\mathbf{M}, \mathbf{C}, \mathbf{K}$ in equation (11) by capacitances, resistances and inductances respectively, and the force vector $\overrightarrow{\mathbf{P}}$ by current sources. Here the problem of negative valued elements also arise, but there are more serious problems. For lossless case, inductor loops may occur and such circuits have to be solved using special techniques. For each non-diagonal entry in the stiffness matrix an inductor is placed into circuit which introduces another unknown (inductor current) in the MNA formulation. If the total number of entries in the stiffness matrix $\mathbf{K}$ is $n \times n$ the circuit matrices in MNA formulation may go up to $\left((\alpha / 2) n^{2}\right) \times\left((\alpha / 2) n^{2}\right)$, where $\alpha$ is the density of sparse stiffness matrix.

In our study the first step is to change the second-order differential equation (11) into a first-order differential equation.

$$
\left[\begin{array}{l|c}
\mathbf{I} & \mathbf{0} \\
\hline \mathbf{0} & \mathbf{M}
\end{array}\right] \cdot \dot{\mathbf{y}}=-\left[\begin{array}{c|c}
\mathbf{0} & -\mathbf{I} \\
\hline \mathbf{K} & \mathbf{C}
\end{array}\right] \cdot \mathbf{y}+\left[\begin{array}{c}
\mathbf{0} \\
\mathbf{P}
\end{array}\right]
$$

where $\mathbf{I}$ is the identity matrix, and

$$
\mathbf{y}=\left[\begin{array}{l}
\overrightarrow{\mathbf{Q}}(t) \\
\dot{\overrightarrow{\mathbf{Q}}}(t)
\end{array}\right]
$$

Equation (23) is similar to the circuit equations (15); but the circuit matrices are positive on the diagonal.

To construct the equivalent circuit we developed two similar methods for the lossless case. These methods are of order $N^{2}$, and the conversion time is very small compared to the simulation time.

The difference of the methods is the choice of the unknowns which corresponds to $\overrightarrow{\mathbf{Q}}(t)$. The extracted circuits have MNA matrices larger than the original FEM problem.

\subsection{Method I}

In the first method, the displacements are chosen as node voltages. Such a choice leads us to choose the velocities as currents through capacitors. This means that there will be one capacitor for each unknown displacement. The other entries are realized using voltage controlled voltage sources and inductors. These are the effects of the displacements in the neighbour elements. The force effects are handled using voltage supplies.

In this method every coupling introduces a series branch, which means an addition of two rows to the circuit matrix; one for the additional node voltage and one for the branch current, so the matrix may become very large.

\subsection{Method II}

Another choice, where the velocities correspond to the node voltages, gives the currents through the inductors as displacements. There is also a capacitor in parallel for each unknown displacement. The other entries are simulated using Current Controlled Current Sources (CCCS). All the circuit elements come in parallel so that no additional equations are needed, but to couple the currents one needs to add zero-volt voltage supplies. The loads at the finite element nodes are realized using current sources. 
For each displacement-velocity pair, there are six rows in the circuit matrix. Although the size of the matrix defining the linear system is six times the original matrix size, the number of entries does not grow much. The number of entries becomes $2 \alpha n^{2}+12 n$ where $\alpha$ is the density of the sparse, $n \times n$, stiffness and mass matrices. So the density of the circuit matrix will be about $2 n^{2} \alpha /(6 n)^{2}=\frac{1}{18} \alpha$.

\section{EXAMPLES}

In this section we will give some examples to demonstrate the speed of the circuit simulation method compared to the FEM solvers. First example is a demonstration of the extraction methods. In other examples, we preferred to use the second method to find the equivalent electrical circuit. The circuits obtained using the first method give the same results.

In these examples, analyses are done using finite element analysis program ANSYS [27] (version 5.3), electrical circuit simulation program HSPICE [3] (version 97.2), and our in house developed electrical circuit simulation program MAWE [7] which uses asymptotic waveform evaluation method with multi-point moment matching. The simulations are performed on a SPARCstation 20 with $50 \mathrm{MHz}$ clock frequency.

\subsection{Example I}

In this example we consider the analysis of a very simple two-mass-spring system shown in Figure 1.

The analysis is done to find the modes of the system. There are only two unknowns ux1 and ux2. In finite element formulation $\mathbf{M}$ and $\mathbf{K}$ matrices are $2 \times 2$.

The equivalent electrical circuits are shown in Figure 2. The upper circuit is obtained using Method I, and the lower circuit is extracted using Method II. In the first circuit the voltages, $v_{c_{1}}$ and $v_{c_{2}}$ across the capacitors $\mathbf{C 1}$ and $\mathbf{C 2}$ correspond to the unknown displacements ux1 and ux2, respectively. The circuit has six node equations and six branch current equations, so the equivalent circuit matrix is $12 \times 12$. In the second circuit the currents, $i_{\mathrm{L} 1}$ and $i_{\mathrm{L} 2}$, through the inductors L1 and $\mathbf{L} 2$ correspond to the unknown displacements. This circuit has also six equations for the node voltages and six equations for the branch currents, and the matrix of the equivalent circuit is again $12 \times 12$.

In finite element analysis the natural frequencies are found to be 2.5814 and $8.3263 \mathrm{~Hz}$. The equivalent circuits have poles at exactly the same frequencies. For this very simple example, the analysis times for the finite element solver and the circuit simulator MAWE are comparable $(0.25$ and $0 \cdot 13 \mathrm{~s}$ ). Only one expansion point is enough as it is a small circuit.

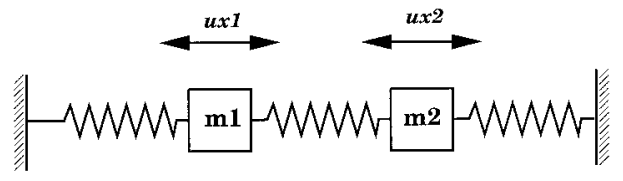

Figure 1. Two-mass-spring system 

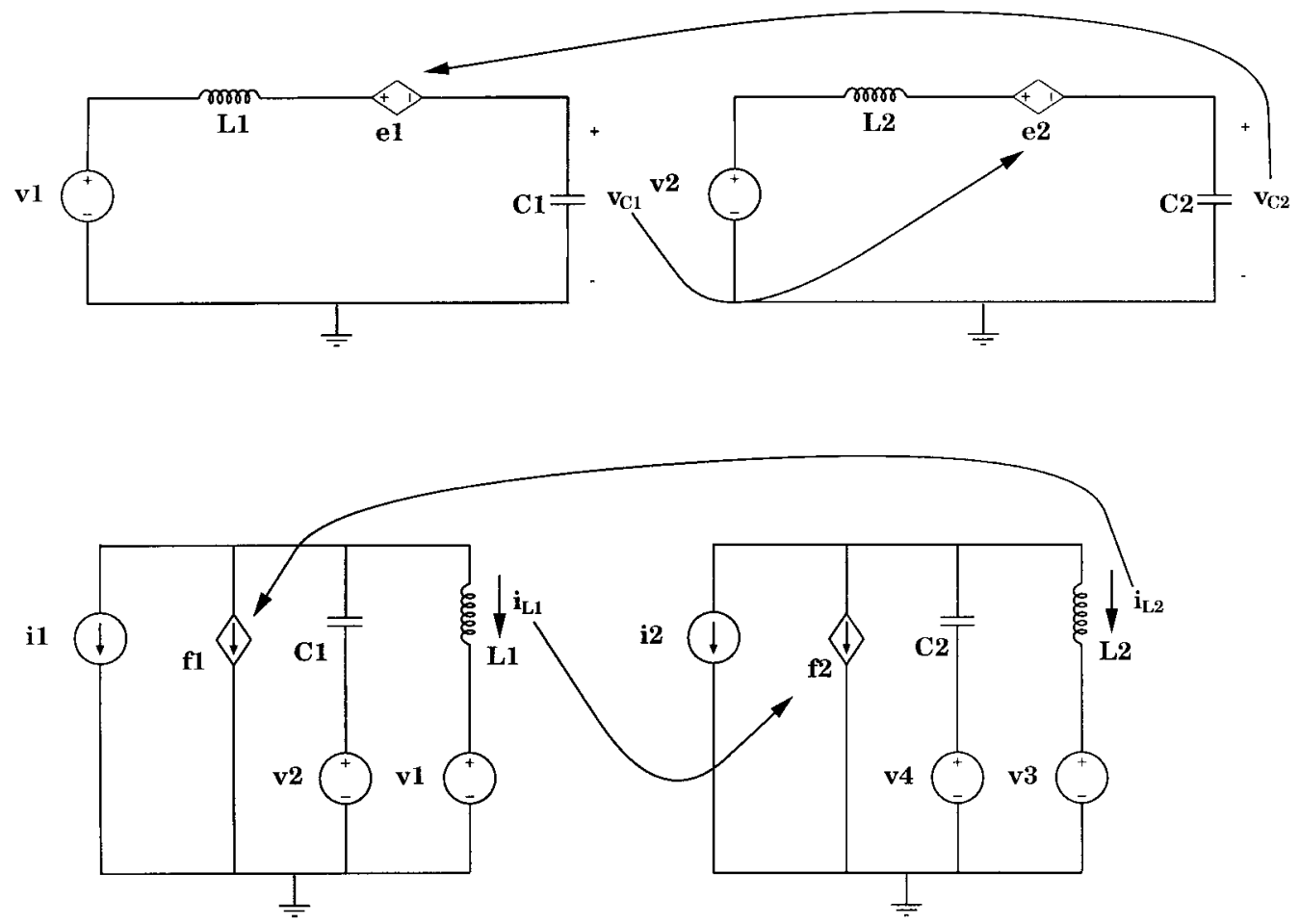

Figure 2. Equivalent circuit for the two-mass-spring system

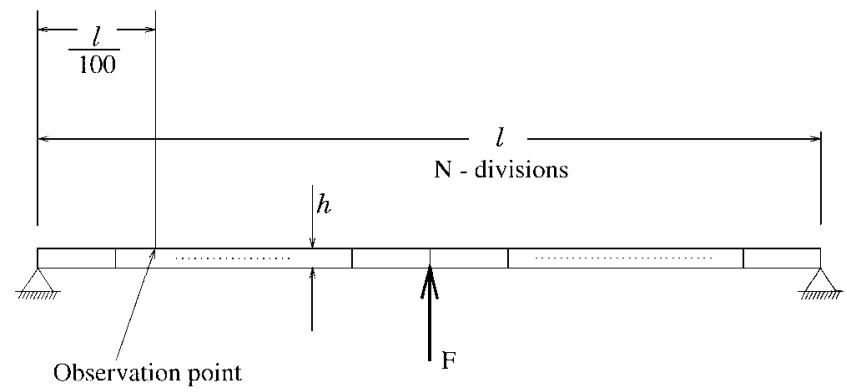

Figure 3. Bar supported at the two sides (Example II)

\subsection{Example II}

This example is the harmonic analysis of a simple bar. We have divided the bar into $N$ elements, apply the force at the mid-point and observe the displacement at 1/100 of the length (Figure 3 ). The system has $N-1 \mathrm{UY}$ unknowns (displacements in $y$-direction) and $N+1$ ROTZ unknowns (rotations around the $z$-axis), which gives a total of $2 N$. The summary of extracted electrical circuit is given in Table II. 
Table II. Circuit summary for Example II

\begin{tabular}{lccccccc}
\hline$N$ & $\begin{array}{c}\text { Number of } \\
\text { unknowns }\end{array}$ & $L$ & $C$ & CCCS & VS & $\begin{array}{c}\text { Number of } \\
\text { circuit nodes }\end{array}$ & $\begin{array}{c}\text { Circuit } \\
\text { matrix size }\end{array}$ \\
\hline 100 & 200 & 200 & 200 & 1788 & 400 & 601 & 1201 \\
200 & 400 & 400 & 400 & 3628 & 800 & 1201 & 2401 \\
400 & 800 & 800 & 800 & 7138 & 1600 & 2401 & 4801 \\
\hline
\end{tabular}

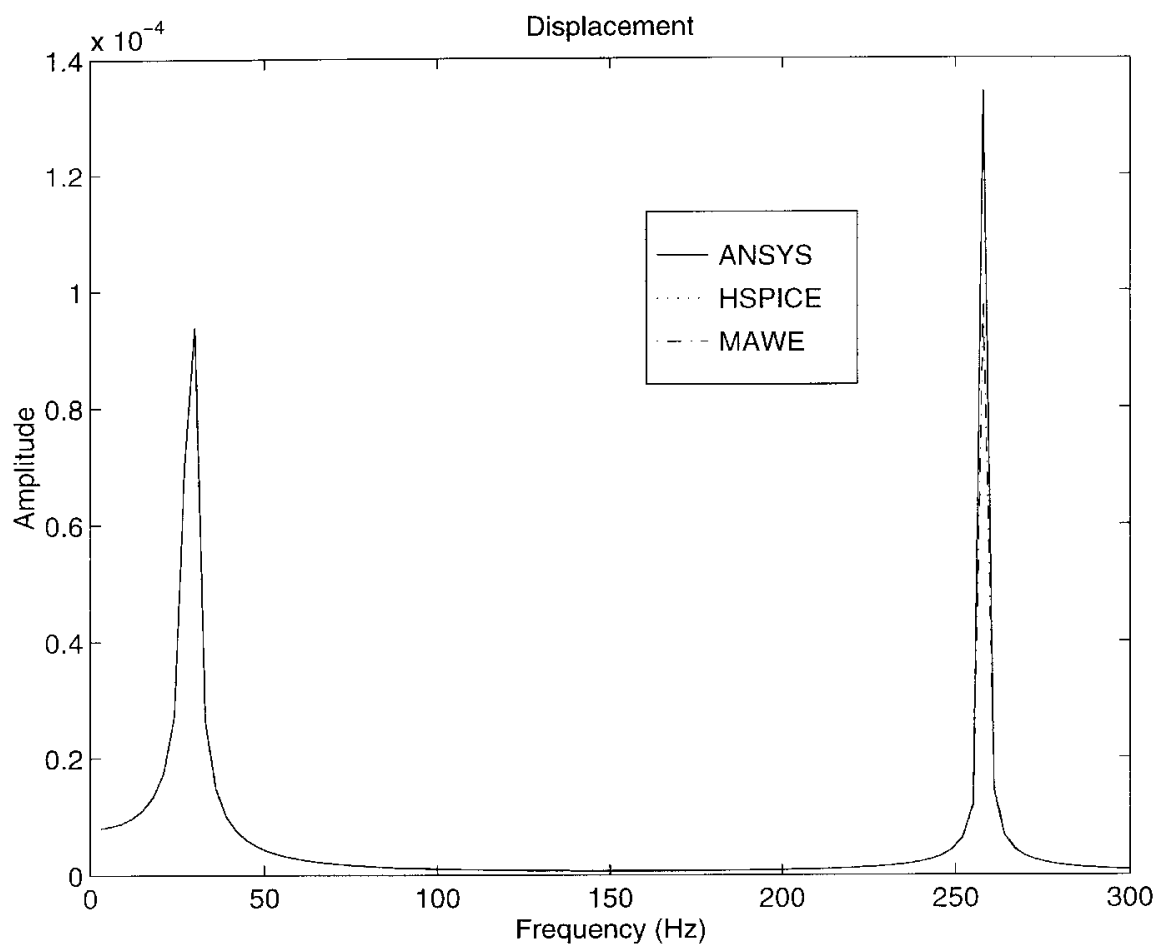

Figure 4. Harmonic analysis results for Example II

It can be seen in Figure 4 that the harmonic response outputs are indistinguishable. In Table III, execution times during the harmonic analyses are shown. (The number of expansion points used in MAWE simulation is shown in parantheses.) In MAWE analysis 6th-order Padé approximation is used, evaluation point is at $s=0$. Electrical solvers do the same job in less time. As $N$ increases, using electrical solvers becomes even more advantageous. As the body is long and thin the couplings between the displacements are small, and the electrical circuit matrix is sparse. For such problems the speed-up advantage of the circuit theoretical methods is very good.

As the electrical circuit gets larger, HSPICE solution time is better than MAWE. This is probably because the in-house developed sparse matrix solver MAWE is not very efficient for large matrices. Although we use the same electrical circuit netlist file for both HSPICE and MAWE, the circuit reading time is very small in MAWE compared to that in HSPICE. 
Table III. Timing results(s) for Example II

\begin{tabular}{lcccc}
\hline & \multirow{2}{*}{$\begin{array}{c}\text { Number of } \\
N\end{array}$} & \multicolumn{3}{c}{ Execution times } \\
\cline { 3 - 5 } frequency points & ANSYS & HSPICE & MAWE(1) \\
\hline 100 & 100 & 57 & $2 \cdot 28$ & 1.83 \\
200 & 100 & 424 & 4.83 & 6.63 \\
400 & 100 & 4918 & 13.80 & 34.78 \\
\hline
\end{tabular}

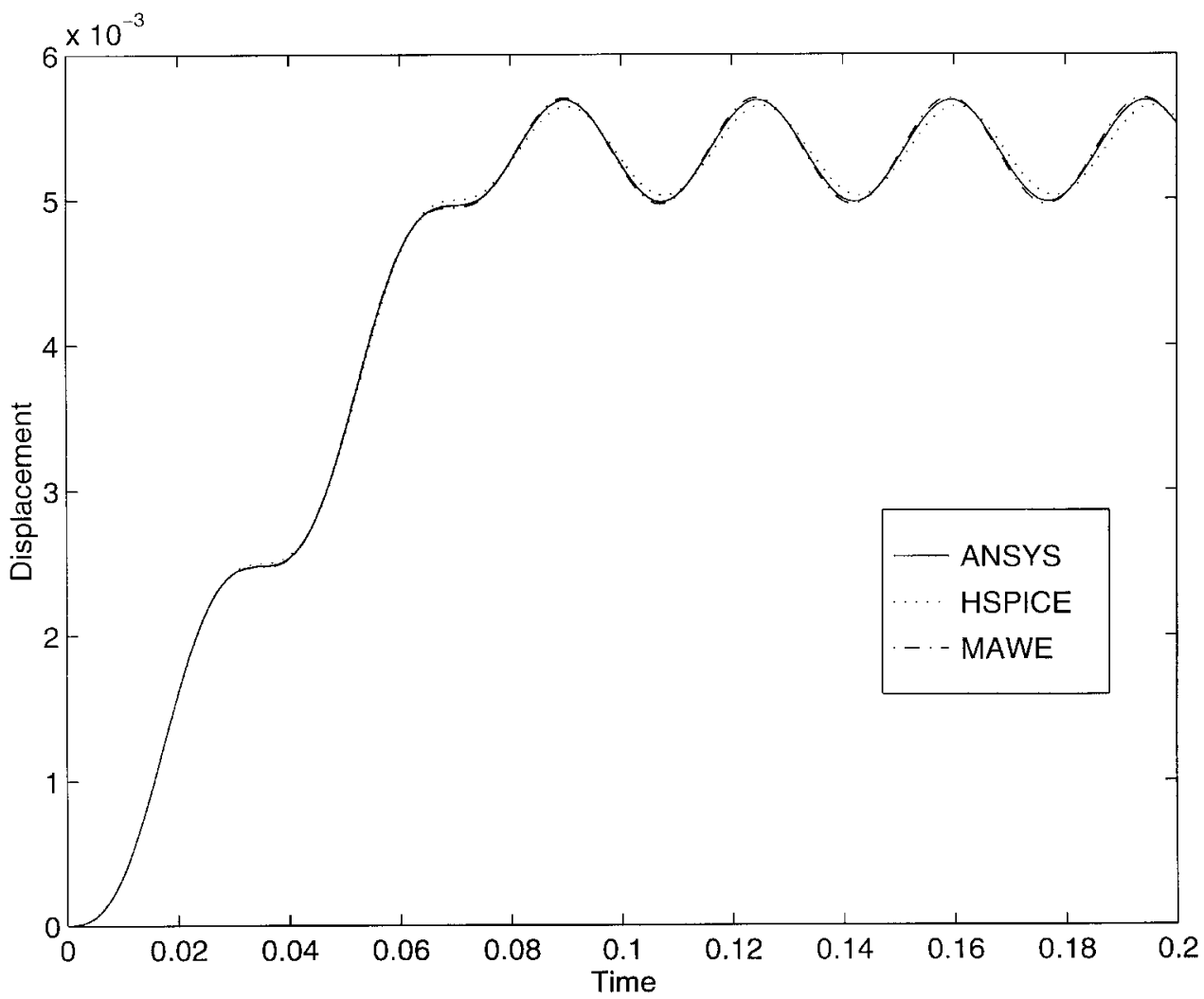

Figure 5. Transient analysis results of the three methods for Example III

In all cases, the output plots are obtained at 100 frequency points. It should be noted that increasing the data points in ANSYS causes a linear increase in the execution time, while the same increase can be achieved at almost no cost in the case of MAWE.

\subsection{Example III}

In this example we analyse the transient behaviour of the bar of Example II. Therefore, the equivalent electrical circuit is the same. A step function with a finite rise time is applied to the mid point of the bar (Figure 3), and the behaviour of the same point in time is simulated. For 
Table IV. Timing results(s) of the transient analysis in Example III

\begin{tabular}{lcrcc}
\hline & \multirow{2}{*}{$\begin{array}{c}\text { Number of } \\
\text { time points }\end{array}$} & ANSYS & HSPICE & MAWE(1) \\
\cline { 3 - 5 } & 200 & $8 \cdot 11$ & $8 \cdot 55$ & $1 \cdot 84$ \\
100 & 200 & $76 \cdot 55$ & $18 \cdot 25$ & $7 \cdot 1$ \\
200 & 200 & $686 \cdot 01$ & $39 \cdot 25$ & $35 \cdot 3$ \\
400 & & & & \\
\hline
\end{tabular}

MAWE, Padé approximation is of order six; and the matrix inversion is evaluated only for $s=0$. It can be seen in Figure 5 that all methods give the same results. The execution times are shown in Table IV. Again, as the number of unknowns increase, the advantage of using electrical simulators become more significant. For 200 unknowns the speed-up ratios are 1 and 4.4 for HSPICE and MAWE, and for 800 unknowns ratios become 17.5 and 19.4.

\subsection{Example IV}

In this example, the harmonic analysis of a simply supported thin annular plate has been done (Figure 6). The $x$ and $y$ components of the displacements, and rotations around the $z$-axis are set to 0 , and at the outside boundary the translations in the $z$-direction and rotations around the radial axis are blocked.

The number of elements along radial direction, N1, is selected as five, and the number of elements along circumferential direction, $\mathrm{N} 2$, is chosen to be 32. As a result the number of unknowns is 512. We want to find the harmonic analysis results, when a force is applied at a node at the inside boundary and the frequency response at the same node is observed.

The equivalent circuit has 1537 nodes, 512 inductors, 512 capacitors, 1024 independent voltage sources, 22708 current controlled current sources, and the total matrix size is 3073 .

In 100 point analyses we obtained a rough figure. (Figure 7 shows the zoomed version of the result obtained by ANSYS.) To have a more accurate result we analysed the same circuit using MAWE only by changing the number of frequency points to 1000 . We saw that in 100 point analyses the pole at $15 \cdot 3 \mathrm{~Hz}$ is missing. At each of the expansion points $s=0, s=60 i$ and $s=120 i, 12$ moments were calculated, and the order of the Padé approximation was 30 . In order to locate the pole accurately we increased the number of expansion points by 1 . We performed the 1000 point analysis, used 4 expansion points $s=0, s=60 i, s=90 i, s=120 i$ and at the expansion points calculated $16,8,8,8$ moments, respectively. The order of the approximation became 32 . To compare the results, harmonic analyses of the same frequency region are done by ANSYS, and MAWE. ANSYS requires $1681.5 \mathrm{~s}$ for 400 frequency points, while MAWE requires only $54 \mathrm{~s}$ for 4000 frequency points. The results of the analyses are shown in Figure 8.

The results of the analyses performed for a higher frequency range is shown in Figure 9. Table $\mathrm{V}$ shows the solution times where the numbers of expansion points used in MAWE simulations are shown in parantheses. 


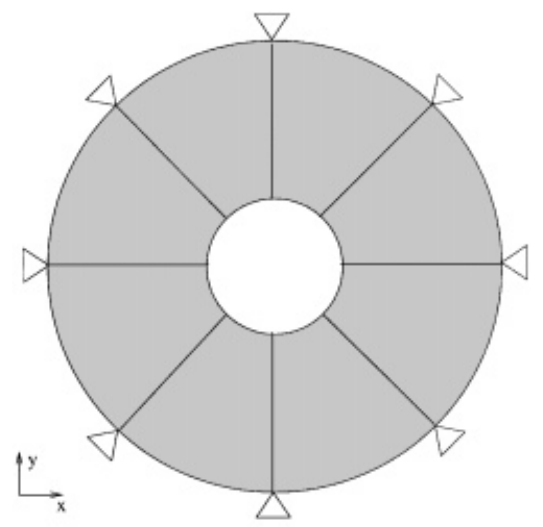

(a)

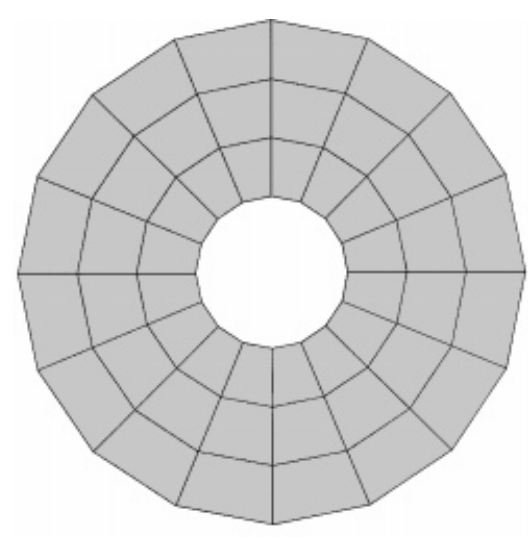

(b)

Figure 6. Simply supported thin annular plate (Example IV)

\subsection{Example $V$}

Fifth example is the harmonic analysis of a solid square plate supported at one edge $(x=0$ plane in Figure 10), force is applied in the $y$-direction at the corner at $(a, a, 0)$. Displacements in the $z$-direction are not allowed, so the displacement has only $x$ and $y$ components.

The equivalent circuit has 1729 nodes, 576 inductors, 576 capacitors, 1152 independent voltage sources, 31726 current controlled current sources, and total matrix size is 3457 .

During the finite element analysis, the body is meshed into $\mathrm{N} 1 \times \mathrm{N} 1$ elements in the $x y$-plane and into $\mathrm{N} 2$ elements in $z$ direction. The problem is reduced to 576 unknowns when the constraints are included. The response is observed at point $(a, 0,0)$. The analyses are done using ANSYS, HSPICE and MAWE. In the first analysis the number of frequency points is 100 . The simulation 


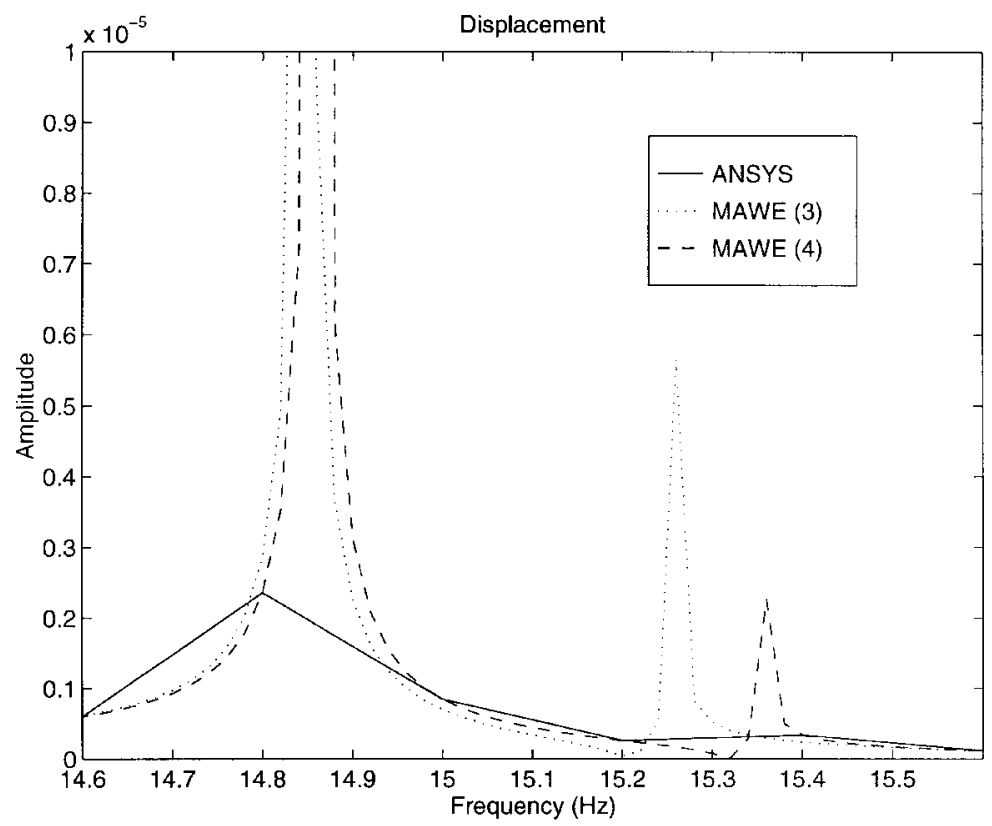

Figure 7. Harmonic analysis results around the missing pole in Example IV

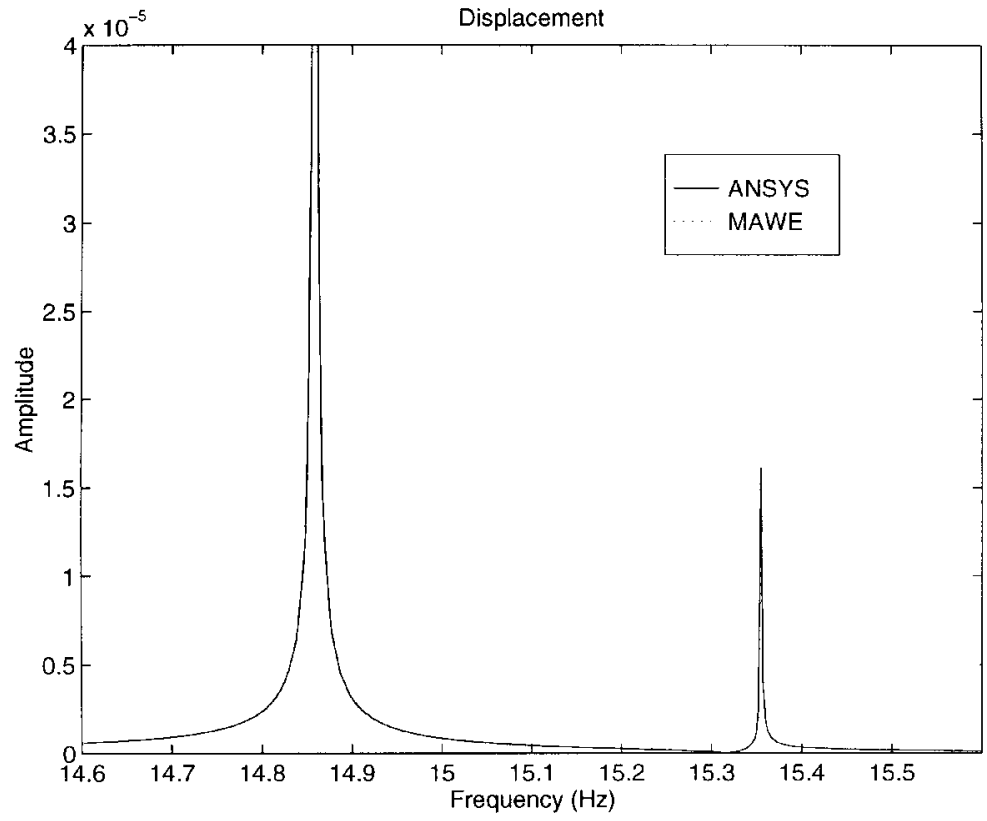

Figure 8. Harmonic analysis results around the missing pole in Example IV 

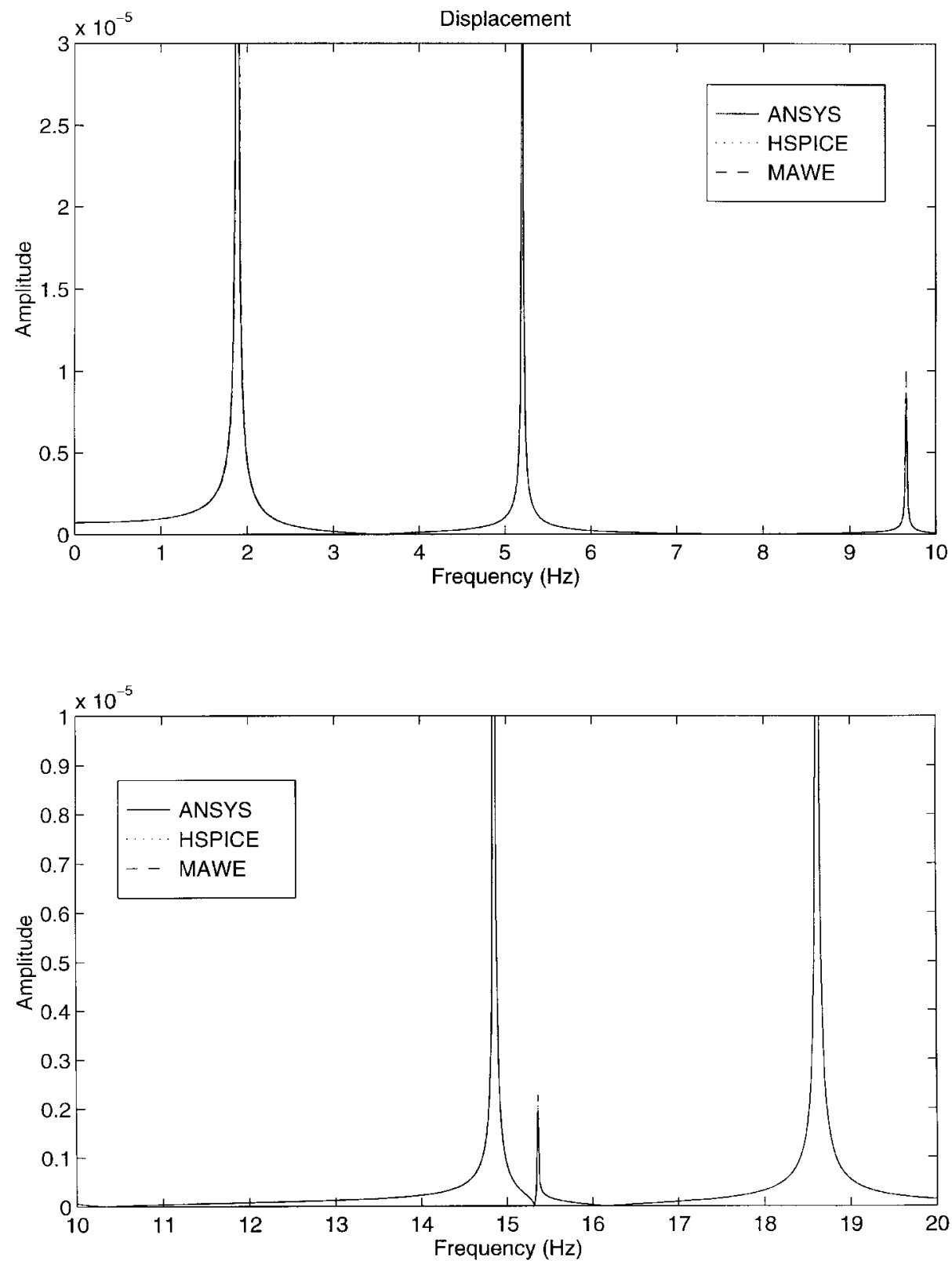

Figure 9. Harmonic analysis results of the three methods for Example IV

results are shown in Figure 11. The order of the Padé approximation is chosen to be 18, in the second analysis 1024 data points are used in HSPICE and ANSYS simulations. Even in the second analysis the results are not accurate and the pole at $132.88 \mathrm{~Hz}$ is missed (Figure 12), so we extended the data point number to 20480 in MAWE simulation with a cost of few seconds 
Table V. Timing results(s) for Example IV

\begin{tabular}{|c|c|c|c|c|c|c|}
\hline \multirow[b]{2}{*}{ N1 } & \multirow[b]{2}{*}{ N2 } & \multirow{2}{*}{$\begin{array}{l}\text { Number of } \\
\text { frequency points }\end{array}$} & \multicolumn{4}{|c|}{ Execution times } \\
\hline & & & ANSYS & HSPICE & MAWE(3) & MAWE(4) \\
\hline 5 & 32 & 100 & $406 \cdot 4$ & $67 \cdot 7$ & $117 \cdot 3$ & $141 \cdot 6$ \\
\hline 5 & 32 & 1000 & 4207 & 561 & $119 \cdot 4$ & $142 \cdot 8$ \\
\hline
\end{tabular}

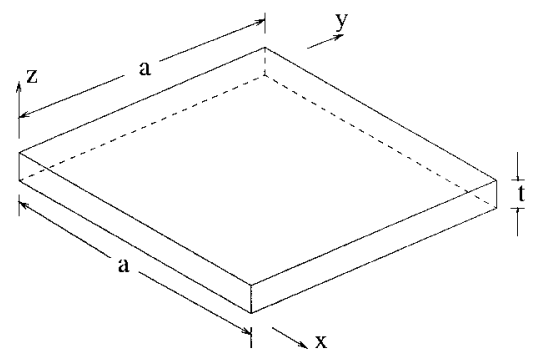

(a)

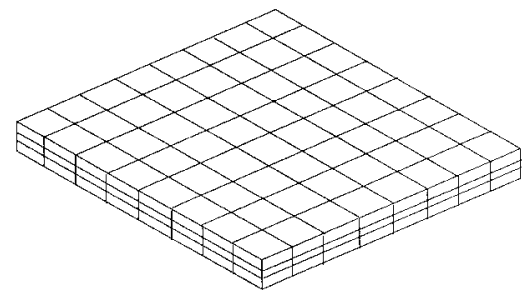

(b)

Figure 10. Solid square plate supported at one edge (Example V)

(increase from $285 \cdot 12$ to $292 \cdot 1 \mathrm{~s}$ ). The moment number-evaluation point pairs used in MAWE simulations are shown in Table VI.

In Table VII, the time consumptions are shown (the numbers of expansion points used in MAWE simulations are shown in parantheses). Again asymptotic waveform evaluation seemed to be the most efficient analysis tool. When the number of data points is increased, the efficiency becomes more apparent.

\section{CONCLUSIONS}

In this paper, the analogy between the finite element formulation of the structural mechanics problems and the electrical circuit theory is investigated. Converting structural mechanics problems 


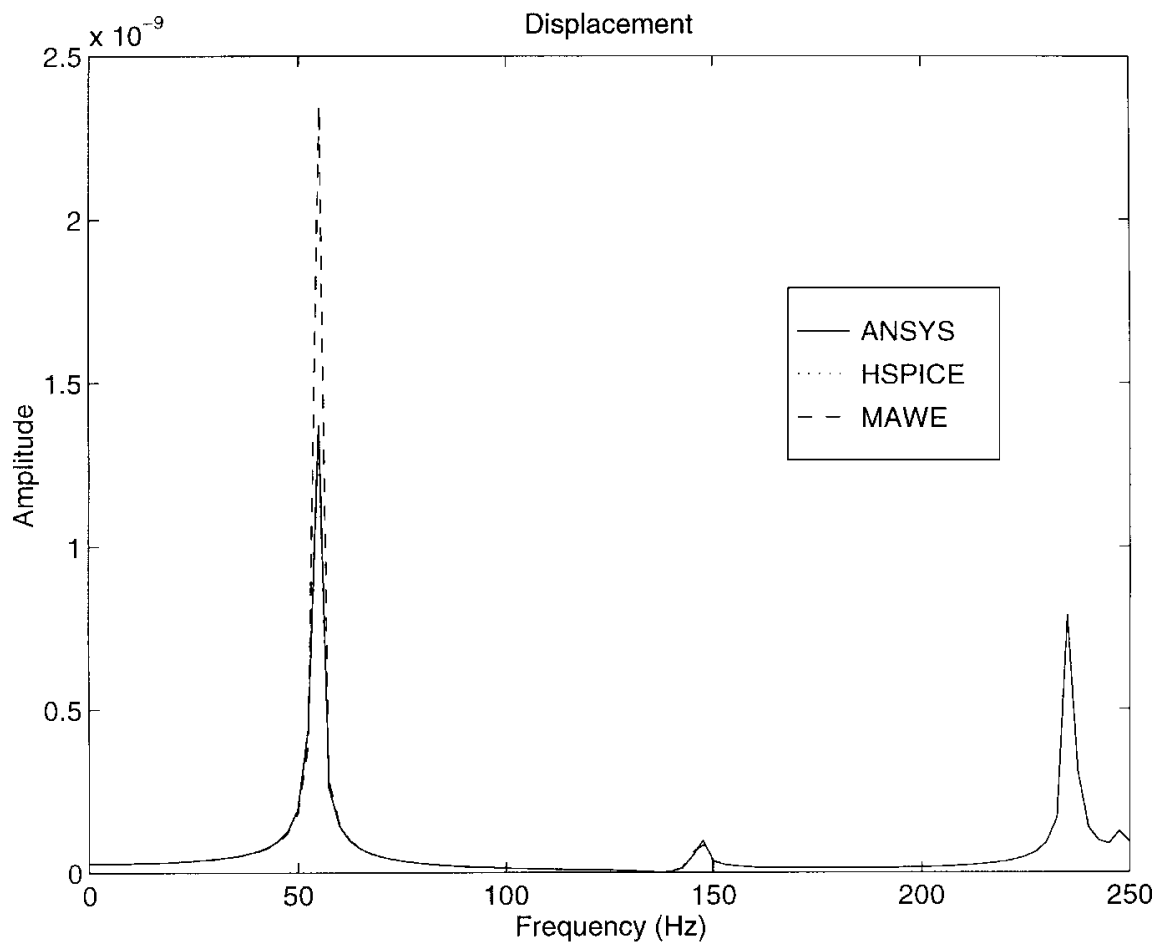

Figure 11. Harmonic analysis results of the three methods for Example V

into circuit analysis problems, we can solve them with a general purpose circuit simulator. Using circuit simulation techniques results in faster solutions for the mechanical problems.

In this work, equivalent circuit extraction is done by a computer program which takes the total mass and stiffness matrices as input and creates an electrical circuit netlist file. In the examples largest netlist is for the last example and the file is built in $1.87 \mathrm{~s}$. The resultant circuit matrix is six times larger, but the required time for the solution can be very small when fast circuit simulation techniques are used.

One method is using moment matching techniques instead of solving the system of equations at each frequency point. The main reason behind the efficiency of these techniques is the decrease in the number of LU decomposition requirements. Multipoint asymptotic waveform evaluation technique requires one LU decomposition per expansion point. Generally, the number of expansion points is much less than the number of frequency points to get an accurate solution. With a good sparse matrix solver, the expected speed-up ratio is almost equal to the number of frequency points divided by the number of expansion points. In this study we needed four expansion points at most. These expansion points are chosen such that they divide the frequency range of interest into equal portions. We stop dividing if the poles of the main system do not change after including the information of the new expansion point.

Previous work on equivalent circuit construction is mainly dealt with coupled field-circuit problems. These studies are mentioned in Section 3. There are two approaches to the problem: 


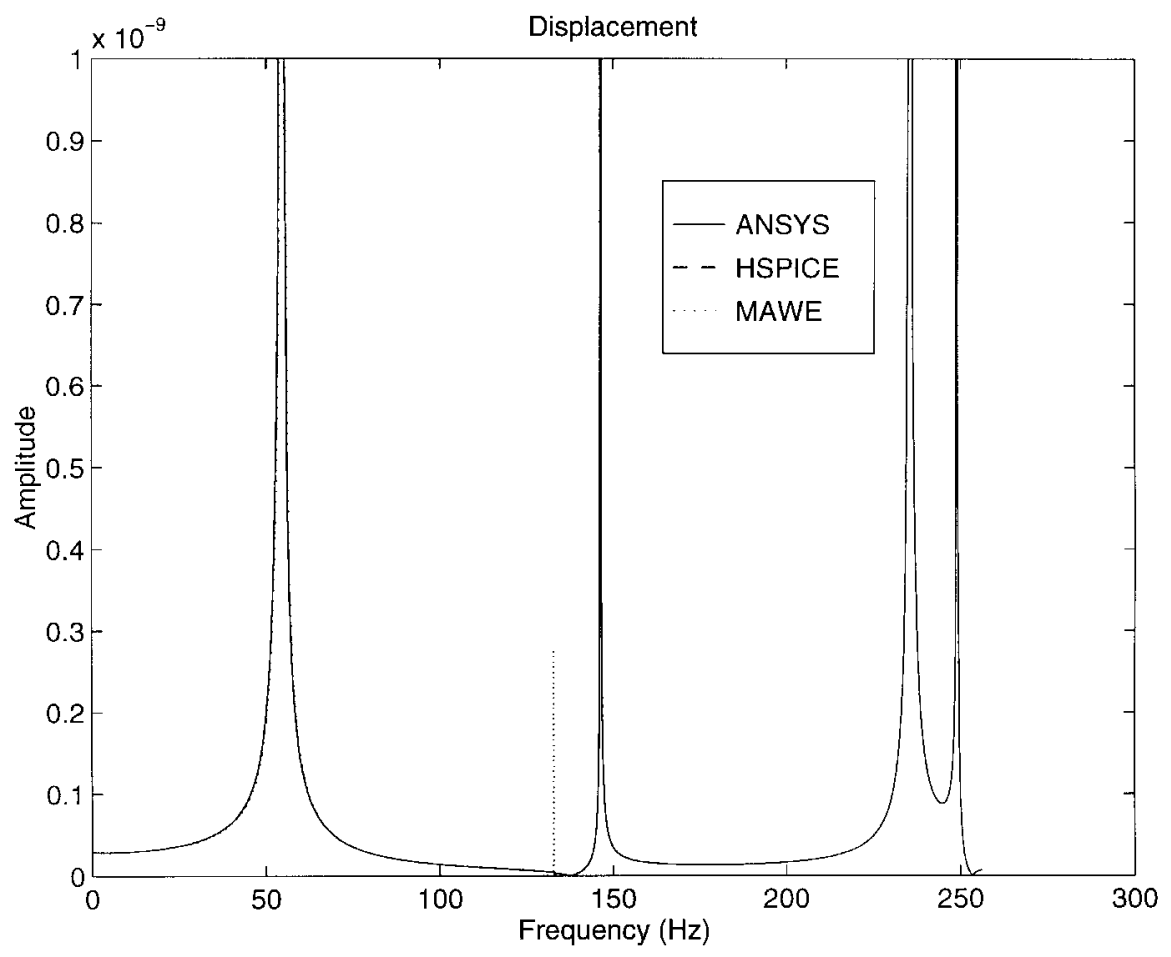

Figure 12. More accurate harmonic analysis results for Example V. The pole at $132.88 \mathrm{~Hz}$ can only be found by MAWE

Table VI. Evaluation point-number of moments pairs (Example V)

\begin{tabular}{lcr}
\hline $\begin{array}{l}\text { Evaluation } \\
\text { point }\end{array}$ & Number of moments \\
\hline 0 & 12 & 16 \\
$800 i$ & & 8 \\
$1200 i$ & 8 \\
$1600 i$ & 12 & 16 \\
\hline Approx. order & 18 & 40 \\
\hline
\end{tabular}

- The part modelled by FEM is solved separately and it is inserted into the circuit as a multiport element.

- Finite elements are converted into elemental equivalent electrical circuits, the whole problem is modelled as an equivalent circuit. The equivalent circuit may have negative valued circuit components, for second-order problems inductor loops may occur, and number of unknowns may grow too much. 
Table VII. Timing results(s) for Example V

\begin{tabular}{lccrrrr}
\hline & & \multirow{4}{*}{$\begin{array}{c}\text { Number of } \\
\text { N1 }\end{array}$} & N2 & Execution times \\
\cline { 4 - 6 } & frequency points & ANSYS & HSPICE & MAWE(2) & MAWE(4) \\
\hline 8 & 3 & 100 & $584 \cdot 7$ & $288 \cdot 8$ & $149 \cdot 4$ & \\
8 & 3 & 1024 & $5986 \cdot 4$ & $2576 \cdot 1$ & $157 \cdot 9$ & 279.9 \\
8 & 3 & 20480 & & & & $291 \cdot 9$ \\
\hline
\end{tabular}

Table VIII. Branch equations for the circuit elements

\begin{tabular}{lcc}
\hline Element & Abbreviation & Branch equation \\
\hline Capacitor & $\mathrm{C}$ & $i_{\mathrm{C}}=\mathrm{C} \cdot \frac{\mathrm{d}}{\mathrm{d} t}\left(v_{\mathrm{n} 1}-v_{\mathrm{n} 2}\right)$ \\
Inductor & $\mathrm{L}$ & $v_{\mathrm{n} 1}-v_{\mathrm{n} 2}=L \cdot \frac{\mathrm{d}}{\mathrm{d} t}\left(i_{L}\right)$ \\
Independent voltage source & $\mathrm{VS}$ & $v_{\mathrm{n} 1}-v_{\mathrm{n} 2}=V$ \\
Independent current source & $\mathrm{IS}$ & $i=I$ \\
Current controlled current source & CCCS & $i=f \cdot i_{\text {cont }}$ \\
Voltage controlled voltage source & VCVS & $v_{\mathrm{n} 1}-v_{\mathrm{n} 2}=e \cdot\left(v_{\mathrm{n} 1 \text { cont }}-v_{\mathrm{n} 2 \text { cont }}\right)$ \\
\hline
\end{tabular}

The objective of our study is to solve the full problem using a general circuit simulator. Our method gives no negative valued electrical circuit components, has replaced the couplings with controlled sources, and can be fully solved with a general purpose electrical circuit simulator, so fast circuit solution methods may be applied easily.

In this work, several examples have been studied using the proposed method and an accurate match with the finite element method results has been obtained. Without a significant loss of accuracy, the simulation speed is improved using moment matching techniques.

Another advantage of these techniques, is that the number of data points can be increased without changing the simulation time significantly, and this will decrease the probability of missing high- $Q$ poles.

The circuits obtained in this study do not have any resistance values, so the circuit matrices are not diagonally dominant. The sparse solvers employed in solving these circuits can be specially designed to achieve better performance.

The methods employed in this work can be generalized to electromagnetic problems. If the semidiscrete equations of the problem have an order higher than two, special care must be taken as this will increase the number of unknowns.

\section{APPENDIX: MODIFIED NODE ANALYSIS FORMULATION}

The simplest way of analysing an electrical linear resistive circuit is to solve for its node voltages with respect to a reference node. Then the branch voltages and branch currents can be calculated easily, if all the elements in the circuit other than current sources are voltage controlled.

In node analysis, the set of equations are obtained by equating the currents entering the nodes to the currents leaving the nodes. The currents are written in terms of the node voltages. 

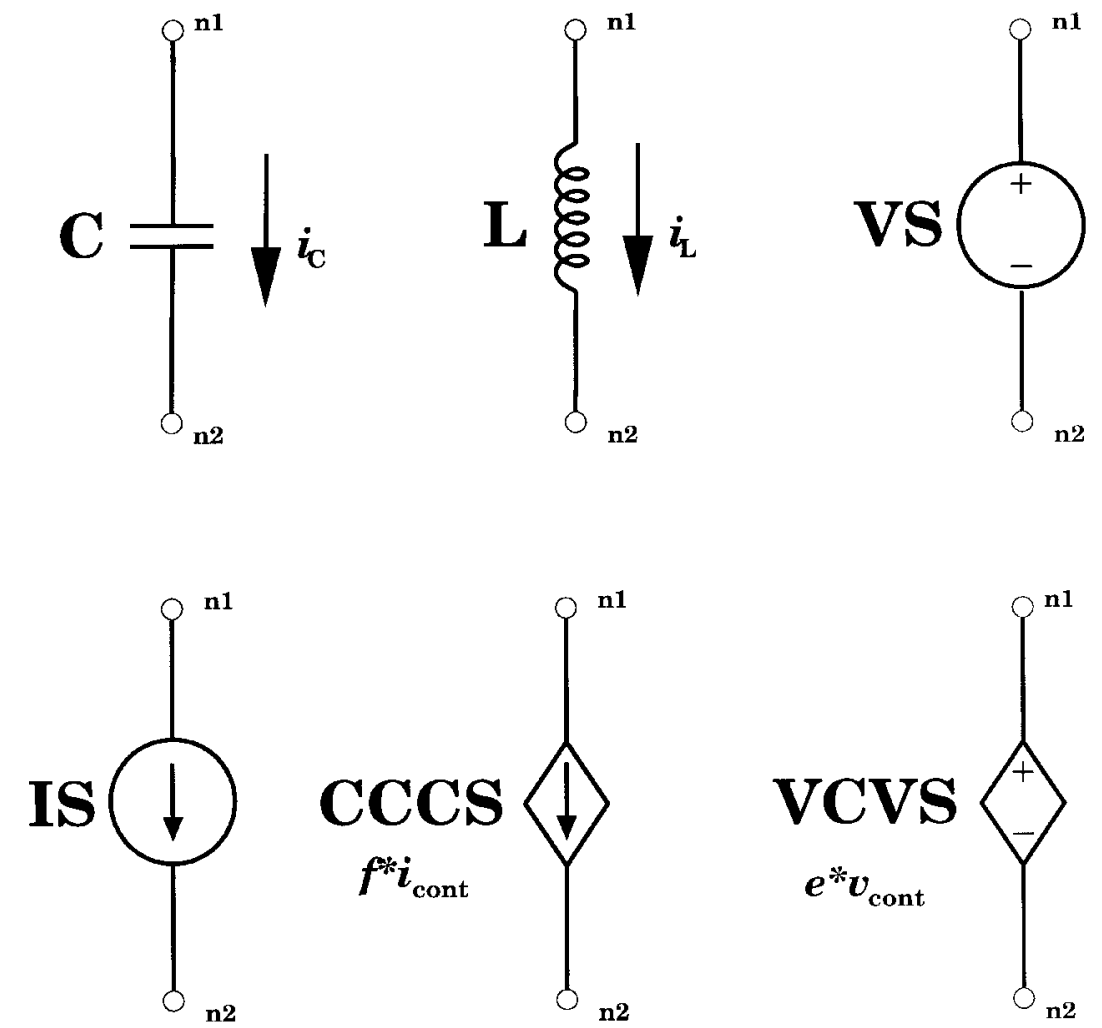

Figure 13. Symbols for the electrical circuit elements

Modified Node Analysis (MNA) is based on node analysis, but it is suitably modified so that it can be used on any electrical dynamic circuit. In MNA the variables used are the node voltages with respect to a reference node and the currents in all branches that are not voltage controlled. The branch equations for these branches are also in the set of equations to be solved. MNA contains small number of unknowns so it is preferred in manual analysis as well as in circuit analysis programs (SPICE, MAWE).

In Table VIII the branch equations, and in Figure 13 the symbols for some electrical circuit components are shown.

\section{REFERENCES}

1. Nagel LW, Pederson DO. Spice (simulation program with integrated circuit emphasis). ERL Memo ERL M382, University of California, Berkeley, April 1973.

2. Nagel LW. Spice2: A computer program to simulate semiconductor circuits. ERL Memo UCB/ERL M75/520, University of California, Berkeley, May 1975.

3. META-SOFTWARE. HSPICE User's Manual. Campbell, CA, 1996.

4. Pillage LT, Rohrer RA. Asymptotic waveform evaluation for timing analysis. IEEE Transactions on Computer Aided Design of Integrated Circuits and Systems 1990; 9:352-366.

5. Chiprout E, Nakhla MS. Analysis of interconnect networks using complex frequency hopping (CFH). IEEE Transactions on Computer Aided Design of Integrated Circuits and Systems 1995; 14(2):186-200. 
6. Celik M, Ocali O, Tan MA, Atalar A. Pole-zero computation in microwave circuits using multi point Pade approximation. IEEE Transactions on Circuits and Systems - I: Fundamental Theory and Applications 1995; 42:6-13.

7. Sungur M, Ekinci AS, Atalar A. An efficient program for analysis of interconnect circuits. International Journal of Electronics 1997; 42:6-13.

8. Lucas TN. Extension of matrix-method for complete multipoint Pade-approximation. Electronics Letters 1993; 29(20):1805-1806.

9. Gabriel K. Equivalent circuits of the elastic field. Journal of Applied Mechanics 1944; 11:A149-A161.

10. Carter GK. Numerical and network analyzer solution for the equivalent circuits for the elastic field. Journal of Applied Mechanics 1944; 11:A162-A167.

11. Jia Tzer Hsu, Loc Vu-Quoc. A rational formulation of thermal circuit models for electrothermal simulation-part I: Finite element method. IEEE Transactions on Circuits and Systems-1: Fundametal Theory and Applications 1996; 43(9):721-732.

12. Jia Tzer Hsu, Loc Vu-Quoc. A rational formulation of thermal circuit models for electrothermal simulation-part II: Model reduction techniques. IEEE Transactions on Circuits and Systems-1: Fundametal Theory and Applications 1996; 43(9):733-744.

13. Tsukerman IA, Konrad A, Menuier G, Sabonnadière JC. Coupled field circuit problems: trends and accomplishments. IEEE Transactions on Magnetics 1993; 29(2):1701-1704.

14. Piriou F, Razek A. Finite element analysis in electromagnetic systems accounting for electric circuits. IEEE Transactions on Magnetics 1993; 29(2):1669-1675.

15. Salon SJ, Ovacik L, Balley JF. Finite element calculation of harmonic losses in ac machine windings. IEEE Transactions on Magnetics 1993; 29(2):1442-1445.

16. Nicolet A, Delincé F, Bamps N, Genon A, Legros W. A coupling between electric circuits and 2d magnetic field modeling. IEEE Transactions on Magnetics 1993; 29(2):1697-1700.

17. Wang JS. A nodal analysis approach for $2 \mathrm{~d}$ and $3 \mathrm{~d}$ magnetic-circuit coupled problems. IEEE Transactions on Magnetics 1996; 32(3):1074-1077.

18. Bedrosian G. A new method for coupling finite element field solutions with external circuits and kinematics. IEEE Transactions on Magnetics 1993; 19(2):1664-1668.

19. Väänänen J, Circuit theoretical approach to couple two-dimensional finite element models with external circuit equations. IEEE Transactions on Magnetics 1996; 32(2):400-410.

20. Shi ZW, Rajanathan CB, A method of approach to transient eddy current problems coupled with voltage sources. IEEE Transactions on Magnetics 1996; 32(3):1082-1085.

21. McDermott TE, Zhou P, Gilmore J. Electromechanical system simulation with models generated from finite element solutions. IEEE Transactions on Magnetics 1997; 33(2):1682-1685.

22. Auld BA. Acoustic Fields and Waves in Solids (2nd edn), vol. I R.E. Krieger Publishing Company: Malabar, FL, 1990.

23. Gong J, Volakis JL. Awe implementation for electromagnetic analysis. Electronics Letters 1996; 32(24):2216-2217.

24. Kolbehdari MA, Srinivasan M, Nakhla MS, Zhang QJ, Achar R. Simultaneous time and frequency domain solutions of em problems using finite element and cfh techniques. IEEE Transactions on Microwave Theory and Techniques 1996; 44(9):1526-1534.

25. Zienkiewicz OC, Taylor RL, The Finite Element Method, Basic Formulation and Linear Problems (4th edn), vol. 1 McGraw-Hill Book Company: London, 1989.

26. Rao SS. The Finite Element Method in Engineering (2nd edn). Pergamon Press: Oxford, 1989.

27. ANSYS, Inc., ANSYS Help System and Documentation, 1995.

28. Greenwood DT. Principles of Dynamics (2nd edn). Prentice-Hall: Englewood Cliffs, NJ, 1988.

29. Chua LO, Desoer CA, Kuh ES. Linear and Nonlinear Circuits, Series in Electrical Engineering. McGraw-Hill: New York, 1987.

30. Tang TK, Nakhla MS. Analysis of high-speed vlsi interconnects using the asymptotic waveform evaluation technique. IEEE Transactions on Computer Aided Design of Integrated Circuits and Systems 1992; 11(3):341-352.

31. Dikmen CT. BUSTLE: A new circuit simulation tool using asymptotic waveform evaluation and piece-wise linear approach. M.S. Thesis, Bilkent University, Department of Electrical and Electronics Engineering, Bilkent, Ankara Turkey, July 1990.

32. Topçu S. PLAWE: A piecewise linear circuit simulator using asymptotic waveform evaluation. Ph.D. Thesis, Bilkent University, Department of Electrical and Electronics Engineering, Bilkent, Ankara, Turkey, July 1994

33. Chiprout E, Nakhla MS. Asymptotic Waveform Evaluation and Moment Matching for Interconnect Analysis. Kluwer: Dordrecht, 1994.

34. Ocalı O, Tan MA, Atalar A. A new method for nonlinear circuit simulation in time domain: Nowe. IEEE Transactions on Computer Aided Design of Integrated Circuits and Systems 1996; 15(3):368-374.

35. Alaybeyi M. BUSTLE: A new circuit simulation tool. M.S. Thesis, Bilkent University, Department of Electrical and Electronics Engineering, Bilkent, Ankara, Turkey, July 1990.

36. Analogy Inc., Beaverton OR. 